\title{
Spherical thermal waves in laser plasmas
}

\section{J. L. Montañes and J. R. Sanmartin}

Escuela Tecrica Superior de Ingenieros Aeronauticos, Universidad Politêcnica de Madrid, Madrid, Spait. (Received 8 November 1977; accepted 20 September 1979)

The thermal wave produced in a uniform plasma, when energy is absorbed on a spherical surface such that convection is negligible, is analyzed using an integral method, which is very accurate. The curvature speeds up (slightly slows down) the inner (outer) wave front and does not affect the temperature maximum.

Local deposition of energy in fluids with nonlinear heat conduction may give rise to thermal waves and negligible convection. ${ }^{1}$ In particular, when energy per unit area and time $\phi=\phi_{0} t / \tau$ is deposited in a given plane within a plasma, where the conductivity is $K$ $=\bar{K} T^{5 / 2}, \bar{K} \approx$ const, a thermal wave develops if $\alpha$ $\equiv\left(9 k / 4 m_{i}\right)\left(k^{2} \tau n_{0}^{2} / \phi_{0} \bar{K}\right)^{2 / 3}$ is small $\left(T, n_{0}, m_{i}\right.$, and $k$ are electron temperature and density, ion mass, and Boltzmann's constant, respectively) ${ }^{2,3}$; if $\alpha$ is too small, however, the conductivity will be less than classical. ${ }^{3}$ In laser-plasma applications, radiation absorption may be limited to a thin, spherical layer. For a monotonically growing pulse of length $\tau$ and maximum intensity $\phi_{0}$, where

$$
\phi=\phi_{0} g(t / \tau), \quad g(1)=1, \quad(0<t<\tau),
$$

a spherical wave should develop for a broad class of pulse shapes and moderately small $\alpha$. Converging thermal waves also appear in other problems and have recently provoked interest, but have been studied very little..$^{4,5}$

For simplicity, consider a uniform plasma and absorption at $r=R$; then, the electron energy equation is

$$
\frac{3}{2} n_{0} k \frac{\partial T}{\partial t}=\frac{\bar{K}}{r^{2}} \frac{\partial}{\partial r}\left(r^{2} T^{5 / 2} \frac{\partial T}{\partial r}\right)+\phi_{0} g(t / \tau) \delta(r-R),
$$

where $\delta$ is Dirac's function. Introducing the dimensionless variables ${ }^{3}$

$$
\begin{aligned}
& \hat{t}=t / \tau, \quad \hat{x}=(r-R) / \epsilon R, \quad \hat{T}=T\left(2 \phi_{0}^{2} \tau / 3 k n_{0} \bar{K}\right)^{-2 / 9}, \\
& \epsilon=\left(2 \tau / 3 k n_{0}\right)^{7 / 9} \bar{K}^{2 / 9} \phi_{0}^{5 / 9} R^{-1},
\end{aligned}
$$

Eq. (1) becomes

$$
\frac{\partial \hat{T}}{\partial \hat{t}}=\frac{1}{(1+\epsilon \hat{x})^{2}} \frac{\partial}{\partial \hat{x}}\left((1+\epsilon \hat{x})^{2} \hat{T}^{5 / 2} \frac{\partial \hat{T}}{\partial \hat{x}}\right)+g(t) \delta(\hat{x}) .
$$

The initial and boundary conditions are

$$
\hat{T}=0 \text { at } \hat{t}=0, \quad \hat{T}=\hat{T}^{5 / 2} \frac{\partial \hat{T}}{\partial \hat{x}}=0 \text { at } \hat{x}=\hat{x}_{f},
$$

$\hat{x}_{f}$ being either the inner $\left(\hat{x}_{\text {in }}\right)$ or outer $\left(\hat{x}_{\text {out }}\right)$ wave front; it may be shown that $\hat{T} \sim\left(1-\hat{x} / \hat{x}_{f}\right)^{2 / 5}$ near $\hat{x}_{f}$ (Ref. 1 ). In addition, the last term in (2) may be dropped if use is made of the condition

$$
\left.\hat{T}^{5 / 2} \frac{\partial \hat{T}}{\partial \hat{x}}\right|_{0^{-}} ^{0^{+}}=-g(\hat{l}) \text {. }
$$

For short times, the curvature effects are small. Expanding in powers of $\hat{t}$ and setting $g=\hat{t}^{\hat{p}}$, we find to second order (quasi-planar approximation)

$$
\hat{x}_{f}= \pm \hat{t}^{(7+5 p) / 9} \xi_{1}+\hat{t}^{(14+10 p) / 9} \xi_{2},
$$

$$
\hat{T}=\hat{t}^{(2+4 p) / 9} \Theta_{1}(s) \pm \hat{t}^{1+p} \Theta_{2}(s), \quad s \equiv \hat{x} / \hat{x}_{f} ;
$$

the upper (lower) sign should be used for $\hat{x}>0$ and $\hat{x}_{\text {out }}\left(\hat{x}<0\right.$ and $\left.\hat{x}_{\text {in }}\right)$. We notice that the planar (lowestorder) solution is symmetric, while the second-order correction is antisymmetric. An excellent approximation to $\xi_{1}$ and $\theta_{1}$ (well known for $\left.p=1\right)^{3,6}$ is

$\xi_{1}=(4 / 5)[7 / 8(1+p)]^{7 / 9}, \quad \Theta_{1}=[7 / 8(1+p)]^{2 / 9}(1-s)^{2 / 5} ;$

both $\Theta_{2}\left[\right.$ which behaves roughly as $\left.s(1-s)^{2 / 5}\right]$ and $\xi_{2}$ are negative. Thus, to lowest order, curvature effects speed up $\hat{x}_{\text {in }}$ and slow down $\hat{x}_{\text {oul }}$, do not affect $\hat{T}(\hat{x}=0)$, and make the inner wave more steplike [the term $2 \bar{K} r^{-1} T^{5 / 2} \partial T / \partial r$ in (1) is positive for $\left.\hat{x}<0\right]$.

We now determine $\hat{x}_{\text {in }}, \hat{x}_{\text {out }}$, and $\hat{T}(\hat{x}=0)$ for arbitrary times. Multiplying Eq. (2) by $(1+\epsilon \hat{x})^{a}$ and integrating between $\hat{x}_{\text {in }}$ and $\hat{x}_{\text {out }}$, we get

$$
\begin{aligned}
& \frac{d}{d \hat{t}} \int_{\hat{x}_{\mathrm{in}}}^{\hat{x}_{\mathrm{out}}}(1+\epsilon \hat{x})^{\mathrm{q}} \hat{T} d \hat{x} \\
& \quad=\frac{2}{7} \epsilon^{2}(q-1)(q-2) \int_{\hat{x}_{\mathrm{in}}}^{\hat{x}_{\mathrm{out}}}(1+\epsilon \hat{x})^{q-2} \hat{T}^{7 / 2} d \hat{x}+g ;
\end{aligned}
$$

for $q=1$ and $q=2$, we arrive at

$$
\begin{aligned}
& \int_{\hat{x}_{\text {in }}}^{\hat{x}_{\text {out }}}(1+\epsilon \hat{x}) \hat{T} d \hat{x}=\int_{0}^{\hat{t}} g\left(\hat{t}^{\prime}\right) d \hat{t}^{\prime}, \\
& \int_{\hat{x}_{\text {in }}}^{\hat{x}_{\text {out }}}(1+\epsilon \hat{x}) \hat{x} \hat{T} d \hat{x}=0 .
\end{aligned}
$$

We then try an asymmetric profile

$$
\begin{aligned}
& \hat{T}(\hat{t}, \hat{x})=\hat{T}_{a}(\hat{l})\left[1-\hat{x} / \hat{x}_{\text {oul }}(\hat{t})\right]^{2 / 5}, \quad 0<\hat{x}<\hat{x}_{\text {out }} \\
& \hat{T}(\hat{t}, \hat{x})=\hat{T}_{a}(\hat{t})\left[1-\hat{x} / \hat{x}_{\text {in }}(\hat{t})\right]^{2 / 5}, \quad \hat{x}_{\text {in }}<\hat{x}<0,
\end{aligned}
$$

which behaves properly near either wave front, and goes over to the planar profile (5) when $\hat{t} \rightarrow 0\left(\hat{x}_{f} \rightarrow 0\right)$. The three unknown functions of $\hat{t}$ may be obtained by using Eqs. (7), (8), and (3). Defining $\sigma \equiv \hat{x}_{\text {out }} /\left(-\hat{x}_{\mathrm{in}}\right)$, we arrive at an implicit equation for $\sigma(\hat{t}, \epsilon)$

$$
\begin{aligned}
\epsilon^{9 / 7} \frac{\int_{0}^{\hat{t} g d \hat{t}^{\prime}}}{g^{2 / 7}} & =G(\sigma) \\
& =\left(\frac{17(1-\sigma)}{1-\sigma+\sigma^{2}}\right)^{9 / 7} \frac{(1+\sigma)^{5 / 7} \sigma^{2 / 7}}{7 \times 2^{11 / 7}}\left(1-\frac{17}{24} \frac{(1-\sigma)^{2}}{1-\sigma+\sigma^{2}}\right),
\end{aligned}
$$

and then obtain $\hat{x}_{\mathrm{out}}, \hat{x}_{\mathrm{in}}$, and $\hat{T}_{a}$ parametrically

$$
\hat{x}_{\text {out }}=-\sigma \hat{x}_{\text {in }}=\frac{17}{10} \epsilon^{-1} \frac{\sigma(1-\sigma)}{1-\sigma+\sigma^{2}}=\frac{2}{5} \frac{1+\sigma}{g(\hat{t})} \hat{T}_{a}^{7 / 2} .
$$




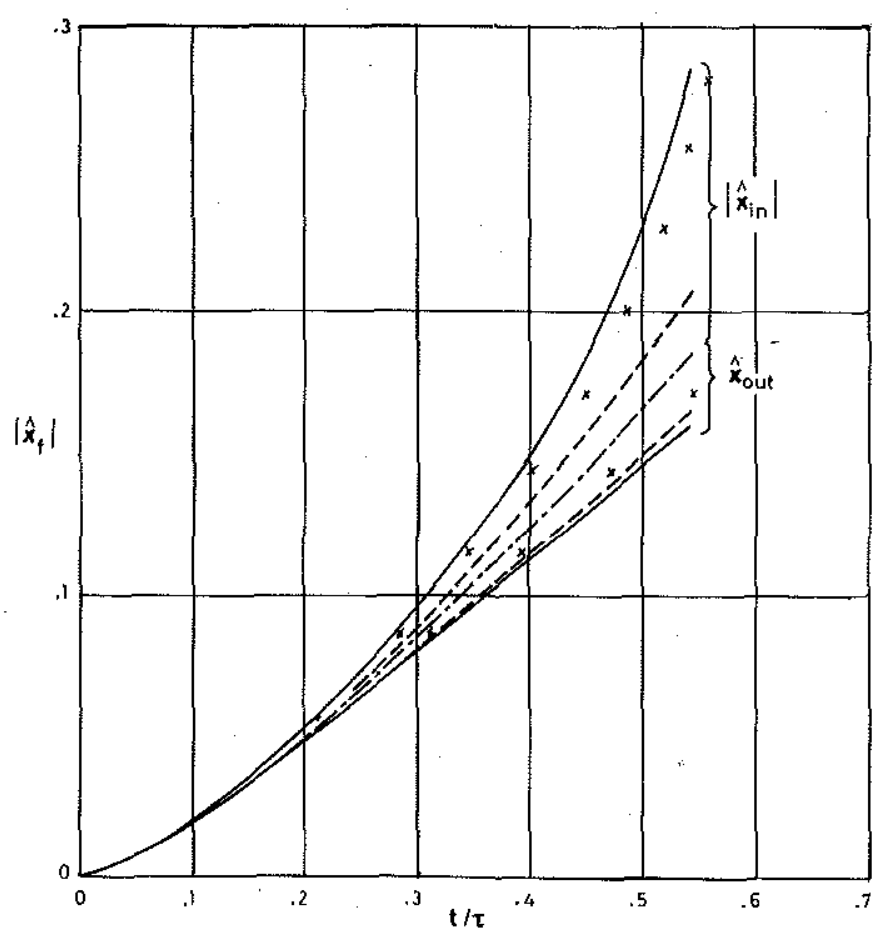

FIG. 1. Dimensionless wave-front positions versus time for a linear pulse $\phi=\phi_{0} t / \tau$, from the integral method (-) (up to the time of arrival of the inner front at the center), and from an exact numerical computation $(x)$, for a 3,5 value of the dimensionless parameter $\epsilon$; also shown are the planar $(-\cdot-)$ and quasi-planar (-- ) approximations.

For a certain $\epsilon$, call it $\epsilon^{*}$, the inner front arrives at the origin $\left(\hat{x}_{\mathrm{in}}=-\epsilon^{-1}\right)$ when the pulse ends $(\hat{t}=1)$. From Eqs. (9) and (10), we get $\sigma\left(\epsilon \hat{x}_{\mathrm{in}}=-1\right) \simeq 0.557$, and

$$
\epsilon^{*}=\left(\frac{0.878}{\int_{0}^{1} g d \hat{t}}\right)^{7 / 9}
$$

as $\hat{t}$ increases, $G$ decreases, going from zero at $\sigma=1$ to 0.878 at $\sigma \simeq 0.557$. For $\epsilon>\epsilon^{*}$, the inner front reaches the origin at a time $\hat{t}_{0}<1$, given by

$$
\left[g\left(\hat{t}_{0}\right)\right]^{-2 / 7} \int_{0}^{\hat{t}_{0}} g d \hat{t}=\left(\frac{\epsilon^{*}}{\epsilon}\right)^{9 / 7} \int_{0}^{1} g d \hat{t} .
$$

For $\epsilon<\epsilon^{*}$, the solution (9) and (10) breaks down when the pulse ends, at a value $\sigma_{1}>0.557$ :

$$
G\left(\sigma_{1}\right)=0.878\left(\epsilon / \epsilon^{*}\right)^{9 / 7} \text {. }
$$

in laser-fusion applications (for future high-energy pulses requiring no compression), one should consider a plasma sphere of radius $R$ in a vacuum, instead of a uniform plasma, as initial conditions; it seems that for such a problem a value of $\epsilon$ not far from $\epsilon^{*}$ should be selected.

A discussion of the results, and their accuracy, is simpler for power law pulses, $g=\hat{t}^{p}$. For $\hat{T}_{a}$ we get

$$
\hat{T}_{a}=[7 / 8(1+p)]^{2 / 9} \hat{t}^{(2+4 p) / 9} F(\sigma),
$$

which differs from the planar (and quasi-planar) result in the factor $F$, an algebraic function lying between 1 at $\sigma=0$ and 1.027 at $\sigma \simeq 0.557$. For comparison we carried out a numerical computation ${ }^{7}$ for $p=1(\epsilon * \simeq 1.55)$ and $\epsilon \simeq 3.5$, yielding $\hat{T}_{a}(\hat{t})$ slightly (less than $1 \%$ ) above the planar values. We conclude that the integral method predicts $\hat{T}_{a}(\hat{t})$ very accurately, and that the curvature does not affect $\hat{T}_{a}$. For the fronts we get

$$
\hat{x}_{\text {out }}=-\sigma \hat{x}_{\mathrm{in}}=\frac{4}{5}\left(\frac{7}{8(1+p)}\right)^{7 / 9} \hat{t}^{(7+5 \phi) / 9}\left(\frac{1+\sigma}{2} F^{7 / 2}\right) ;
$$

they are shown in Fig. 1 for $p=1$ and $\epsilon=3.5$, together with the planar and quasi-planar results. Also shown are numerical values for the same conditions. Clearly, (i) the integral method predicts the front positions accurately, and (ii) the curvature speeds up $\hat{x}_{\text {in }}$ and slightly slows down $\hat{x}_{\text {out }}$. We verified, in the exact, numerical solution, that the curvature steepens (flattens) the inner (outer) wave profile, although neither one is far from the (approximate) planar profile, $\left(1-\hat{x} / \hat{x}_{f}\right)^{2 / 5}$.

We notice that the integral method does not work in cylindrical geometry, for which only the $q=1$ moment of the energy equation takes a simple form.

This research was performed under the auspices of the Junta de Energia Nuclear of Spain.

${ }^{1}$ Ya. B. Zel' dovich and Yu. P. Raizer, Physics of Shock Waves and High-Temperature Hydrodynamics Phenomena (Academic, New York, 1966), Vol. II, Chap. X.

${ }^{2}$ S. I. Anisimov, Zh. Elksp. Teor. Fiz. Pis' ma Red. 12, 414 (1970) [JETP Lett. 12, 287 (1970)].

${ }^{3}$ A. Barrero and J. R. Sanmartin, Phys. Fluids 20, 1155 (1977).

${ }^{4}$ V. P. Korobeinilkov, Trudy Mat. V. A. Steklov Univ. 119, Akad. Nauk SSSR (1973).

${ }^{5}$ E. A. Berchenko and V. P. Korobeinikov, Dokl. Akad. Nauk SSSR 230; 1306 (1976) [Sov. Phys. -Dokl. 21, 557 (1977)].

${ }^{6}$ R. C. Mjolsness and H. M. Ruppel, Phys. Fluids 15, 1620 (1972).

${ }^{7}$ C. Bonacina and G. Comini, Int. J. Heat Mass Transfer 16, 581 (1973). 